Frith \& Done avoid this in part by flaunting the convention of classical phenomenology and attributing hallucinations to thoughts, rather than treating them as percepts. If they do accept hallucinations as percepts then they themselves would place this phenomenon in a category entirely separate to actions, and rightly so, since the notion of 'willing' a percept is not convincing. (One may will an image, which is why percepts and images are entirely distinct experiences.) Is a belief an 'action'? A belief is a predisposition to act. The associated mental 'action' is the thought which is used to rehearse the belief. Is an emotion an 'action'? Anyone who has been unable to change their feelings but has been able to change their actions could describe the difference, and would say without doubt that emotion is most definitely not the result of 'willed intent'.

I would therefore suggest that physical actions and thoughts do have a willed intention, although this is rarely conscious outside of situations of conflict. However, percepts, beliefs, and emotions are not produced by willed intention - other than those intentions of which we are as unconscious as the schizophrenic person. These irreducible mental events are different both from actions and from each other. The Frith \& Done theory thus succeeds only as a neuropsychology for the abnormal schizophrenic experiences of the passivity phenomena of thoughts and actions, and the willed intention to think, i.e. Gedankenlautwerlen. (The only experimental evidence cited is in fact for the monitoring of motor actions by schizophrenic patients.) It is not convincing for the entirely different experiences of percepts, beliefs, and feelings.

If such events are not willed, where do they come from and how do they arise? We must assume that percepts arise from sensory experiences, and that beliefs and emotions arise from the black box of our consciousness as a consequence of other inputs. They do so in a way that we can make some sense of -i.e. in an 'understandable' fashion. Thus we feel justified in labelling these events, as we do, as our own. Here Dr Frith points out (in my view correctly) that we differ in this way from the schizophrenic person who sometimes fails to achieve correct 'labelling' of these events. I would suggest that this is simply because these events appear to arise de novo, not because they are a consequence of unmonitored intentions. They could appear to arise de novo because they occur as a consequence of a structural or biochemical abnormality, or because they arise from one's consciousness in an non-understandable way - presumably for the same reasons. Given their non-understandable origin, these events will sometimes be recognised as 'not of self' (i.e. not arising from one's own con- sciousness). They will then be (incorrectly) attributed to externally generated sensations, producing hallucinations, or to external agents, producing the passivity phenomena of sensations and feelings. They could also be attributed to thoughts and actions, allowing one to abandon the faulty internal monitor altogether. Sometimes they will be accepted as "of self', resulting in delusions which are accorded the same special status as religious insights: not arising from data from the outside world, and therefore not requiring testing against it. If 'will' or 'willed intention' is yet another mental event, there is no reason why these should not also arise apparently de novo in the schizophrenic person, thus creating conflicting wishes and intentions, and the ambivalence of the will.

Such a hypothesis draws directly on the theories of Hughlings Jackson: any cerebral lesion results in negative symptoms, due to loss of tissue, and to positive symptoms, since loss of tissue results in the 'release' of other areas of the brain. It is both more parsimonious than that offered by Drs Frith \& Done, and in line with the traditional phenomenological and philosophical understanding of such mental events. It explains how pathological, physiological, and biochemical changes at almost any level of the central nervous system can result in psychotic phenomena of any sort, in any illness, simply because they can produce these mental events. It seems superfluous to invoke a final common pathway through an 'internal monitor'. Schizophrenia may well be a distinct illness, but it is not its capacity to cause psychotic phenomena that makes it distinctive.

\section{York Clinic}

FrANCES KLEMPERER

\section{Guy's Hospital}

London SEI 9RT

SIR: I would like to add to the model of Drs Frith \& Done by putting forward some ideas derived from my interest in artificial cognition. Hofstadter (1982) has pointed out that thought has a monitoring quality with increasing levels of abstraction and breadth of observation. He likened this to a mathematical regression, but pointed out that the regression is not infinite as the self-watching nature of a monitor at some level of abstraction detects infinite, or even mildly repetitive, patterns of thought. The lowest level of monitoring would be the conscious channel through which our thoughts run, analogous to a central processing unit (mainly processing, little abstraction), and the equivalent of the comparator of Gray (1981). I propose changing the schema illustrated by Drs Frith \& Done in their Fig. 1 by suggesting that 
willed intention and stimulus intention are themselves monitoring units, but with a higher level of abstraction. My model would have a number of levels of monitoring, progressing upward from conscious thought, through willed intentions, on through plans and ideas to the most abstract of concepts. My way of illustrating this would be to suggest that as one progresses up to higher levels of monitoring, the process or energy of thought ceases to have a sequential characteristic like an action potential sweeping along an axon, and becomes a deforming force acting on the plastic boundaries of concepts, like a huge Venn diagram in which the lines are driven to break, merge, and reform. At some level the nature of thought, and the nature of a concept, with all its fluidity, become inseparable.

I believe that the lesions in schizophrenia lie at a higher level than Drs Frith \& Done suggest, in the feedback and feedforward mechanisms of a specific subsystem. My model could be used to account for the variability in clinical manifestations and complete psychopathology of schizophrenia. Frith (1987) described negative symptoms as a response to positive symptoms, but I believe Crow's (1980) concept of two separate constellations of symptoms, type I and type II, which may or may not coexist, has validity. I propose that the specific subsystem affected in schizophrenia has a specialised function in rapidly monitoring perceptions, actions, and thoughts personally or directly affecting the organism. This subsystem has a powerful feedback and feedforward channel to and from conscious thought, and is organised at all levels, but its boundaries become less clear as one moves up from conscious sequential thought towards concepts relating to the self. The most abstract level I can conceive of is that of feelings. It is easy to see the evolutionary advantages conferred by a specialised system constantly alerting us to, and processing, information relating to personal hazard or advantage. The operation of this subsystem within my own brain suggests that it identifies and processes the various aspects of an 'Appening Directly Affecting $\mathrm{Me}$, or ADAM, hence the ADAMs subsystem.

My model of schizophrenia proposes a breakdown in the transfer of information from the lowest level, conscious thought monitor into and out of the ADAMs subsystem. Owing to the increasing blurring of boundaries at higher levels of abstraction, information can enter the subsystem at higher levels (i.e. idea and concept levels), but the information is then more vague, has come through inter-related ideas and concepts, and is not easily identified as being related to an internal or external ADAM.

I postulate that positive symptoms of schizophrenia result from defective feedforward from the
ADAMs subsystem, and negative symptoms from defective feedback into the same subsystem. For example, to act is a concept which activates ideas, plans, and intentions, resulting in information passing through consciousness, and on to the action itself. If the process as perceived by the conscious monitor lacks input from the ADAMs subsystem, then it is experienced as an act with no personal component, and is thus interpreted as control by an outside force. Frith (1987) gave examples of thinking leading to the experience of thought insertion, switching attention leading to delusions of reference, and subvocal thought leading to auditory hallucinations or thought broadcasting. Using my model the equivalent processes occur, but in addition negative and other symptoms can be explained. If the feedback link (from the conscious monitor into the ADAMs subsystem) fails to operate, then perceptions do not activate the cluster of ideas and concepts relating directly to the self. If the highest conceivable level of abstraction within the subsystem is that of feelings, then it can be seen that the likelihood of information reaching this level is diminished, the failure being manifest as poverty of affect. In fact, some information may diffuse into the ADAMs subsystem via the fuzz that surrounds related concepts, but it can be seen that the relationship to the original perception may be tenuous, and even based on the readily available concept of oppositeness. Thus, inappropriate, labile, or shallow affect may be seen as the result. Since it is an important function (for survival itself) of the ADAMs subsystem to direct attention to happenings that might personally affect one, it can be seen that a failure in the direct feedback channel from the conscious stream of thought results in woolly shifts of attention from one vaguely related concept to another, a phenomenon manifesting as thought disorder. Lack of volition and poverty of speech can be seen to follow from a failure of the activating, attention-directing function of the ADAMs subsystem when it is starved of direct input. Presumably even the discomfort resulting from catatonic posturing or exhaustion resulting from catatonic excitement can lack a personal component, and thus fail to activate an appropriate self-preserving response.

Information-processing models of mental illness are probably the models that will be developed and extended in the next few years, and since the development of new models requires conceptual jumps it is impossible to say what impact they may have on our future practice. It is important for us to keep our minds open to new ways of conceptualising the mind and its malfunctions; we must be prepared to delve into the psychological insights of researchers 
working in the superficially dry fields of information processing and artificial cognition.

Brunswick House

Sue Adams

299 Glossop Road

Sheffield S10 $2 \mathrm{HL}$

\section{References}

Crow, T. J. (1980) Molecular pathology of schizophrenia. More than one dimension of pathology? British Medical Journal, 200, 66-68.

FRITH, C. D. (1987) The positive and negative symptoms in schizophrenia reflect impairments in the perception and initiation of action. Psychological Medicine, 17, 631-648.

GrAY, J. A. (1981) The Neuropsychology of Anxiety. Oxford: Oxford University Press.

HofstadTER, D. (1982) Variations on a theme as the essence of imagination. Scientific American, 247, 14-21.

\section{Self-rating}

SIR: McLaren (Journal, November 1988, 153, 708) regards an individual's rating of himself (as either good or bad) as irrational and self-defeating. Certainly the concept of overall or global self-esteem does not make sense in the context of contemporary society. However, it does make sense in the context of evolutionary biology (Lancet, 1988), and it may help us to lead people away from the harmful tendency to rate themselves globally if we understand why this self-evaluative behaviour might have evolved.

The development of high or low global self-esteem may be seen as a preparation for crucial situations in which decisions have to be made between selfassertion and self-effacement. High global selfesteem is a strategy for self-assertion, low global self-esteem a strategy for self-effacement.

It is likely that the capacity for both high and low self-esteem is present in everybody at birth, the selection of strategy being made at predetermined stages. Crawford (1987) made a useful distinction between developmentally contingent strategies, in which the selection is made early in life, long before the strategy has to be 'played', and concurrently contingent strategies, in which the selection is affected by factors operating at the time of the 'play'. In human beings it seems likely that the level of self-esteem which determines the decision between self-assertion and self-effacement in any adult situation is both developmentally and concurrently contingent.

A developmentally contingent low self-esteem strategy may be inculcated by parents in one or more of their offspring (by withholding praise or administering punishment) as part of an evolutionary parental strategy in its own right; or it may be imposed by other adults, such as teachers, as a form of inverse nepotism; or by older siblings to whom it may be an advantage for younger siblings to be predisposed to self-effacement; or it may be selected in the selfevaluative rough-and-tumble of childhood or the adolescent peer-group. These individuals, who are familiar to us in our clinical work, enter and endure adult life with chronic low self-esteem which is often very resistant to treatment.

In other patients we can identify a concurrently contingent low self-esteem strategy in the form of a depressive state. Some people respond to stress with elevation of mood, giving them enhanced vigour and competence associated with a rise of self-esteem; others respond with a reduction of mood, giving them reduced vigour and competence associated with lowered self-esteem. Either strategy is effective at dealing with the prototypical interpersonal stress situation in which two equal adversaries are in competition and neither will give way - a situation which we share not only with other primates but with all other vertebrates. In humans the environmental factors will be more complex than in animals, and will almost certainly involve the acquisition or loss of allies, so the proximate causation may well be one of loss rather than of manifest competition.

The view of depression as a concurrently contingent low self-esteem strategy is not in conflict with other causative theories of depression, whether they be psychoanalytical, behaviouristic, neurophysiological, or biochemical; nor is calling depression a strategy in conflict with its conceptualisation as disease, reaction, or posture (Hill, 1968). It is merely adding the perspective of ultimate (evolutionary) causation to those methods which seek to analyse proximate (immediate) causation.

\section{Milton Keynes General Hospital} Milton Keynes MK6 SLD

\section{References}

CRAWFORD, C. B. (1987) Sociobiology: of what value to psychology? In Sociobiology and Psychology (eds C. B. Crawford, M. Smith \& D. Krebs). Hillsdale, NJ: Lawrence Erlbaum Associates. LANCET (1988) Self-esteem. Lancet, ii, 943-944.

HILL, D. (1968) Depression: disease, reaction, or posture? American Journal of Psychiatry, 125, 445-457.

\section{Pre-pabertal Depressive Stupor}

SIR: I read with interest the case report of prepubertal depressive stupor (Journal, November 1988, 153, 689-692). I reported a similar case several years ago (Warneke, 1975). This patient was first admitted in a depressive stupor at the age of 12 years. He was initially admitted to a pediatric service where 